\title{
Effects of Nigella sativa on glycemic control, lipid profiles, and biomarkers of inflammatory and oxidative stress: A systematic review and meta-analysis of randomized controlled clinical trials
}

\author{
Jamal Hallajzadeh $^{1}$ (i) | Alireza Milajerdi $^{2,3}$ | Moein Mobini ${ }^{4}$ | Elaheh Amirani ${ }^{5}$ | \\ Susan Azizi $^{6}$ | Elhameh Nikkhah ${ }^{7}$ | Babak Bahadori ${ }^{7}$ | Razieh Sheikhsoleimani ${ }^{8}$ | \\ Seyyed Mehdi Mirhashemi ${ }^{9}$ (i)
}

${ }^{1}$ Department of Biochemistry and Nutrition, Research Center for Evidence-Based Health Management, Maragheh University of Medical Sciences, Maragheh, Iran

${ }^{2}$ Students' Scientific Research Center, Tehran University of Medical Sciences, Tehran, Iran

${ }^{3}$ Department of Community Nutrition, School of Nutritional Sciences and Dietetics, Tehran University of Medical Sciences, Tehran, Iran

${ }^{4}$ Faculty of Kinesiology, University of Calgary, Calgary, Alberta, Canada

${ }^{5}$ Research Center for Biochemistry and Nutrition in Metabolic Diseases, Kashan University of Medical Sciences, Kashan, Iran

${ }^{6}$ Student Research Committe, Maragheh University of Medical Sciences, Maragheh, Iran

${ }^{7}$ Medicinal Plants Research Center, Maragheh University of Medical Sciences, Maragheh, Iran

${ }^{8}$ Student Research Committee, Qazvin University of Medical Sciences, Qazvin, Iran

${ }^{9}$ Metabolic Diseases Research Center, Research Institute for Prevention of NonCommunicable Diseases, Qazvin University of Medical Sciences, Qazvin, Iran

Correspondence

Seyyed Mehdi Mirhashemi, Metabolic

Diseases Research Center, Research Institute for Prevention of Non-Communicable

Diseases, Qazvin University of Medical

Sciences, Qazvin, Iran.

Email: mirhashemismm@gmail.com

Funding information

Qazvin University of Medical Sciences, Grant/

Award Number: IR.QUMS.REC.1398.306
The aim of this systematic review and meta-analysis was to evaluate the effects of Nigella sativa (N. sativa) on glycemic control, lipid profiles, and biomarkers of inflammatory and oxidative stress. Two independent authors systematically examined online databases consisting of, EMBASE, Scopus, PubMed, Cochrane Library, and Web of Science from inception until October 30, 2019. Cochrane Collaboration risk of bias tool was applied to assess the methodological quality of the studied trials. The heterogeneity among the included studies were assessed using the Cochrane's $Q$ test and $I$-square $\left(I^{2}\right)$ statistic. Data were pooled using a random-effects model and weighted mean difference (WMD) was considered as the overall effect size. A total of 50 trials were included in this meta-analysis. We found a significant reduction in total cholesterol (WMD: -16.80 ; 95\% Cl: $-21.04,-12.55)$, triglycerides (WMD: -15.73; 95\% Cl: -20.77, -10.69), LDL-cholesterol (WMD: -18.45 ; 95\% Cl: -22.44 , -14.94) and VLDL-cholesterol (WMD: -3.72 ; 95\% Cl: $-7.27,-0.18$ ) following supplementation with $N$. sativa. In addition, there was significant reductive effect observed with $\mathrm{N}$. sativa on fasting glucose (WMD: -15.18 ; 95\% Cl: $-19.82,-10.55$ ) and $\mathrm{HbA1C}$ levels (WMD: $-0.45 ; 95 \% \mathrm{Cl}$ : $-0.66,-0.23$ ). Effects of $N$. sativa on CRP (WMD: -3.61 ; 95\% Cl: $-9.23,2.01$ ), TNF- $\alpha$ (WMD: $-1.18 ; 95 \% \mathrm{Cl}:-3.23,0.86$ ), TAC (WMD: 0.31; 95\% Cl: 0.00, 0.63), and MDA levels (WMD: $-0.95 ; 95 \% \mathrm{Cl}$ : $-2.18,0.27)$ were insignificant. This meta-analysis demonstrated the beneficial effects of $\mathrm{N}$. sativa on fasting glucose, HbA1c, triglycerides, total-, VLDL-, LDLcholesterol levels.

\section{KEYWORDS}

HDL-cholesterol, insulin resistance, LDL-cholesterol, meta-analysis, Nigella sativa, oxidative stress 